\section{La dirección y su aporte al desempeño docente en las instituciones de preparación preuniversitaria}

\author{
The directorate and its contribution to teaching \\ performance in pre-university preparation institutions
}

\begin{abstract}
RESUMEN
El propósito de este trabajo es determinar la correlación existente entre la dirección y el desempeño docente en academias preuniversitarias. Se seleccionó una muestra probabilística de 55 docentes. Se desarrolló una investigación de campo no experimental, tipo descriptiva-correlacional de corte transversal o transeccional. Se procedió al análisis de los resultados mediante el programa estadístico IBM SPSS. Para la recopilación de la información se utilizó la técnica de la encuesta y escala tipo Likert. Se obtuvo un resultado de ,624; concluyendo que existe una relación positiva significativa entre dirección y desempeño docente por lo que es importante un buen proceso de dirección para obtener una respuesta positiva de sus docentes mediante el liderazgo, motivación, comunicación, entre otros.
\end{abstract}

Palabras claves: Desempeño docente; dirección; educación.

\begin{abstract}
The aim of this research is to determine the correlation between management and teaching performance in pre-university academies. A sample of 55 teachers was selected. It was developed a non-experimental field work in a descriptive-correlational cross-sectional or transectional method. The results were analyzed by using the IBM SPSS statistical program. To collect the information, the survey technique used a Likert-type scale. A result of, 624 was obtained; concluding that there is a significant positive relationship between leadership and teacher performance and good communication. That is the reason why a good management process is important to obtain a positive response from teachers.
\end{abstract}

Keywords: Teaching performance; direction; education.

\section{Fátima Elizabeth Canales} Torres

elifati.11@gmail.com Universidad Nacional José Faustino Sánchez Carrión 


\section{INTRODUCCIÓN}

En la necesidad de fortalecer los conocimientos recibidos en la educación secundaria (siendo muchas veces deficiente) y buscando ingresar a diversas universidades donde los cupos son limitados, surgen las academias de preparación preuniversitarias. Estas instituciones preparan a los estudiantes para que aprueben el examen propuesto por la Universidad de su preferencia y logren ingresar a una carrera profesional.

Las instituciones educativas de preparación preuniversitaria mantienen una fuerte rivalidad en el mercado, que les exige continuamente mayor eficacia (capacidad de lograr que sus estudiantes a partir de la preparación recibida aprueben el examen de la universidad de su preferencia) y eficiencia (las instituciones de preparación preuniversitaria están obligadas a innovar y mejorar continuamente sus procesos organizacionales, emplear docentes altamente competitivos, así como a utilizar herramientas tecnológicas educativas, entre otros recursos). Estamos en un entorno cada vez más cambiante, lo que implica la necesidad de contar con ventajas competitivas que aseguren su sustentabilidad.

El factor de efectividad está asociado a la relación entre los estudiantes preparados y los que logran ingresar. Por ello, mientras más estudiantes ingresan a las universidades, mayor efectividad de la institución; esto genera mejor imagen y prestigio.

En lo que respecta a la dirección Marciaga (2015) sostiene:

La dirección es un aspecto importante para el desarrollo de las organizaciones porque esto trae como consecuencia el comportamiento del recurso humano de la empresa. Exige un alto nivel de liderazgo de quienes dirigen la empresa, requiere el establecimiento de un sistema de comunicación que permita a los docentes trabajar en equipo, coordinar todos sus esfuerzos y contribuir eficazmente al cumplimiento de los objetivos de la institución. (párr. 1)

Se hace necesario que las academias de preparación preuniversitaria tengan una buena función de dirección basado en valores y equipos de trabajo competentes buscando el crecimiento y desarrollo profesional del docente que contribuyan alcanzar la efectividad.

El desempeño profesional del docente es clave para lograr resultados óptimos en estas instituciones. Muchos estudios realizados por investigadores señalan que un buen proceso de dirección ayuda al mejor desempeño del trabajador, en este caso del docente.

Este trabajo tiene relevancia en cuanto requiere relacionar conocimientos asociados a la dimensión que caracterizan la función de dirección de una institución de preparación preuniversitaria y el desempeño profesional docente para encontrar su correlación y cuyos resultados constituyen en un aporte valioso para mejorar el servicio que ofrecen estas instituciones educativas, entonces, se plantea como objetivo general de la investigación: determinar la correlación existente entre la dirección institucional y el desempeño del docente de las instituciones de preparación preuniversitaria.

Buscando soporte para esta investigación se revisó diferentes documentos científicos y textos de autores reconocidos, así tenemos a Louffat (2015) quien indica "el proceso de dirección vela por las relaciones humanas en la institución" (p. 150). Este proceso de dirección imprime cambios en el ambiente laboral de los colaboradores lo cual es importante tomar en cuenta porque influye en el desempeño del docente; además, el activo más importante en una institución educativa es su capital humano y dentro de esta categoría destaca la importancia del personal docente, siendo clave el desempeño profesional del docente para lograr la misión educativa que tiene la institución.

Algunos antecedentes con referencia a estas variables tenemos: Reyes (2012) en su trabajo de investigación Liderazgo directivo y desempeño docente en el nivel secundario de una institución educativa de Ventanilla - Callao, tuvo como objetivo determinar la relación significativa entre la percepción del liderazgo directivo y el desempeño docente, el método fue descriptivo con una población y muestra de 40 docentes utilizando como instrumento el cuestionario, se concluye que no hay presencia de relación significativa entre la percepción de los 
estilos de liderazgo directivo y el desempeño docente, recomendando un mayor énfasis en el liderazgo distribuido en las escuelas con la finalidad de conseguir el mejoramiento de la calidad educativa en el nivel secundario.

Rodriguez (2017) en su trabajo de investigación El desempeño docente y el logro de aprendizaje en el área de comunicación en las instituciones educativas de Chaclacayo, tuvo como objetivo determinar la relación existente entre el desempeño docente de comunicación con el logro de aprendizaje de los estudiantes del quinto grado de secundaria y mediante el método descriptivo con una población y muestra de 253 estudiantes del 5to grado de Educación Básica del área de Comunicación utilizando como instrumento el cuestionario se concluye que las dimensiones de preparación, enseñanza y participación de los docentes tienen relación con el aprendizaje de los estudiantes del 5to año de educación secundaria de las Instituciones educativas de la localidad de Chaclacayo, recomendando énfasis en competencia comunicativa e importancia a infraestructura, equipos multimedia, talleres de motivación.

Chavez (2017) en su trabajo de investigación Desempeño docente según estudiantes del VII ciclo de instituciones educativas técnicas del distrito de Pangoa - Satipo, tuvo como objetivo establecer las diferencias significativas respecto al desempeño docente según estudiantes del VII ciclo de instituciones educativas técnicas del distrito de Pangoa - Satipo y mediante el método descriptivo con una población y muestra de 275 estudiantes del VII ciclo de instituciones educativas utilizando como instrumento el cuestionario se concluye: que no existe diferencia significativa de frecuencias con respecto a desempeño docente según estudiantes del VII ciclo de instituciones educativas técnicas del distrito de Pangoa - Satipo, recomendando talleres de capacitación para el fortalecimiento del desempeño del docente y mejore la calidad de enseñanza.

Con los mencionados antecedentes podemos entender que una buena dirección orientada al liderazgo, comunicación y trabajo en equipo mejora la satisfacción, el compromiso y el rendimiento de los docentes.

\section{Dirección.}

Es un proceso orientado a dirigir y facilitar las actividades dentro y fuera de la organización, siendo una de las principales funciones en toda organización, al respecto D'Alessio (2015) indica que la dirección debe manejar los aspectos operacionales y estratégicos, para lograr el cumplimiento de la misión de la organización. Louffat (2015) menciona "la dirección es primordial porque se ejecuta y lleva a la práctica con los trabajadores todo lo planeado y organizado, de esta manera los trabajadores se convierten en actores principales" (p. 150). Se hace prioridad para una organización contar con calidad y experiencia en el rol directivo, así como aplicar estilos de liderazgo acorde con la realidad y situaciones de la empresa. Asimismo, Koontz, Wrihrich y Cannice (2012) indican que la función gerencial de dirigir se define como el proceso de influir en las personas para que contribuyan a las metas organizacionales y de grupo. Realizar una dirección adecuada ayuda que estas instituciones educativas de preparación preuniversitaria puedan cumplir con su misión, mejorando su función de docencia, aportando a la productividad y calidad del servicio que prestan.

\section{Liderazgo factor humano de dirección.}

La dirección es una función esencial donde los líderes de la empresa asumen un papel protagónico como agentes de transformación, motivación, orientación y facilitación. Al respecto Fischman (2018) indica que los "líderes deben estimular un ambiente de confianza, que permita que la información fluya" (p. 137). Asimismo, Méndez (2018) indica que el líder debe fomentar una comunicación efectiva, donde las personas expresen opiniones y comentarios sobre la gestión del líder.

En la misma línea, Koontz et al. (2012) describe el liderazgo como el proceso de influir en las personas para que participen con disposición, entusiasmo y confianza hacia el logro de los objetivos del grupo. Se destaca la importancia que tiene el líder para llevar a la organización a la conquista de su visión.

\section{Motivación.}

Chiavenato (como se citó en Arbaiza, 2010) sostiene que "la motivación, las actitudes, la percepción, la personalidad y el aprendizaje son 
procesos básicos. La motivación es uno de los elementos más importantes para comprender el comportamiento de las personas" (p. 152). Resaltando la motivación como un factor primordial para generar buen desempeño, Koontz et al. (2012) declaran que la esencia del liderazgo es tener seguidores.

Arbaiza (2010) precisa, en su relación de la motivación con el desempeño, "motivar a las personas para que alcancen o superen el desempeño esperado, es vital para los gerentes y administradores, en el mundo de los negocios" (p. 151). De lo señalado se aprecia la importancia que tiene para la dirección la motivación, ya que está asociada directamente con el comportamiento del trabajador y su desempeño de la institución.

\section{Relaciones interpersonales.}

Méndez (2018) señala que la organización debe facilitar actividades de carácter social, cultural, deportiva u otras con el fin de fortalecer las interpersonales. Esto afianza la integración y cohesión de las personas con la organización situación que propicia un mejor desempeño del docente.

Los procesos de interacción social dentro de la empresa determinan el nivel de cohesión social, integración y calidad del trabajo en equipo, por lo que, la organización construye identidad alrededor de aspectos tales como valores, ética y responsabilidad social, en cumplimiento de sus políticas y principios (Méndez, 2018). Asimismo, recomienda fortalecer las relaciones en: calidad de la dinámica del trabajo en equipo y en comunicación formal e informal, participación en la solución conjunta a problemas, apego a la ética y principios organizacionales. Para crear las condiciones favorables al surgimiento de lo señalado, la organización cuenta con sus líderes (directores), quienes aplicando sus competencias fomentan la excelencia en el trabajo en equipo, la comunicación, la cooperación, la formación en valores, entre otras. Esto impacta positivamente la calidad de las relaciones interpersonales.

\section{Comunicación.}

Para todas las funciones y procesos que requiere la organización es esencial la comunicación en su afán de alcanzar la visión. Al respecto,
Koontz et al. (2012) manifiestan que "una estrategia de comunicación es esencial para la ejecución apropiada de las metas corporativas y el desarrollo y mantenimiento de la marca de una empresa y su ventaja competitiva" (p. 471), sin duda la comunicación asume un valor estratégico para las organizaciones, así, Betancourt (2019) declara "Disponer de una estrategia comunicacional efectiva constituye... un factor clave de éxito dada la dinámica de los cambios en el entorno externo e interno que afectan el desempeño organizacional, siendo necesario evaluar y controlar cualquier desviación ocasionada por estos cambios" (p. 79), entonces de esta cita se infiere la necesidad de la comunicación para atender lo que acontecen en el ámbito interno y externo de la organización.

En lo que respecta a la comunicación interna Fischman (2018) indica que las personas necesitan siempre más información, en consecuencia, las empresas deben realizar un plan de comunicación permanente utilizando diferentes recursos y estrategias, tales como reuniones periódicas, boletines informáticos, se hace necesario entender que cuando los empleados están desinformados se genera falta de confianza y desmotivación lo que origina efectos negativos al ambiente de trabajo y por ende el desempeño laboral.

\section{Reconocimiento, beneficios sociales.}

Una de las principales motivaciones para las personas es el reconocimiento, en ese sentido Fischman (2018) señala que el reconocimiento debe ser una herramienta fundamental de formación de cultura y mejora del desempeño.

Es importante la frecuencia con que se hace el reconocimiento, por tal motivo algunos autores proponen que debe ser parte de la cultura ya que contribuye a un mejor desempeño, así, Fischman (2018) menciona la necesidad de establecer un sistema de reconocimiento formal, que según investigaciones se evidencia que aquellas personas que hacían una buena comunicación del reconocimiento lograban que el 95\% de los empleados se sintieran reconocidos realmente, contra solo el 38\% si la comunicación no era adecuada.

En cuanto a los beneficios sociales, Koontz et al. (2012) indican que pueden lograr que el 
rendimiento de una empresa en cuanto a trabajo y tiempo se logren con grandes expectativas. Algunas empresas suelen utilizar beneficios como: vales de ticket para la comida y/o gasolinera, ayuda con estudios, reconocimiento por ejemplo el empleado del mes, entre otros.

Contar con un recurso humano competente y alineado a los objetivos estratégicos es esencial para toda empresa puesto que le permite competir en el mercado esto conlleva a que la empresa debe seleccionar personal acorde a las exigencias en materia de competencias técnicas o profesionales, además de planificar acciones de adiestramiento para mantener actualizado a su personal.

\section{Desempeño profesional del docente.}

Los docentes son un factor clave en la búsqueda de una educación de calidad. Al respecto la Organización de las Naciones Unidas para la Educación, la Ciencia y la Cultura (2013) refiere que “...el pilar fundamental de la calidad educativa son las capacidades profesionales docentes: los alumnos deben tener en sus aulas docentes capaces de generar mayores oportunidades de aprendizaje, caso contrario, no se producirá un genuino mejoramiento de la calidad educativa" (p. 25).

Asimismo, la Ley № 28044, Ley General de Educación, en el literal e del artículo 13, dispone incentivar el desarrollo profesional y el buen desempeño laboral ya que es uno de los factores que interactúa para el logro de la calidad de la educación. Desde la perspectiva profesional debe considerarse al docente como actores de cambio, de él depende la mejora en el proceso de enseñanza ya que el análisis teórico del desempeño pedagógico del docente se comprende como el proceso que vincula las competencias didácticas con la experiencia práctica acumulada, teniendo en cuenta la dimensión humana. De esta manera, Montenegro (2007) manifiesta que "el desempeño del docente es el cumplimiento de sus funciones; el cual se encuentra determinado por factores docente, estudiante y entorno" (p. 32). Asimismo, Chiroque (2006) dice que "el desempeño docente se refiere a las prácticas que ejercen los docentes, en relación a las obligaciones inherentes a su profesión y cargo" (p. 65).
En la misma línea, Díaz (2006) indica que el desempeño docente es lo que debe de hacer, demostrar y reflejar en el aula de clase teniendo en cuenta la tecnología, la planificación curricular, las estrategias didácticas, los materiales didácticos y la evaluación que medirá los resultados en el proceso de enseñanza - aprendizaje. De ahí la importancia de definir estándares para el proceso de evaluación al docente que debe de estar contemplado en el ejercicio del proceso de dirección, que ayuden para que este brinde una enseñanza de calidad, de esta manera será un actor destacado en el desarrollo del ecosistema del conocimiento.

\section{MÉTODOS}

Se realizó la construcción de un diseño metodológico que permita la descripción y análisis de las variables objeto de estudio. Hernandez-Sampieri y Mendoza (2018) señalan que la investigación es "conjunto de procesos sistemáticos, críticos y empíricos que se aplican al estudio de un fenómeno con el resultado de ampliar su conocimiento" (p. 4). La investigación se desarrolla según el enfoque cuantitativo.

La investigación es de campo y con nivel descriptivo; como lo señala Hernández, Fernández y Baptista (2006), consiste en "analizar el nivel de una o diversas variables en un momento dado" (p. 186). El diseño de la investigación es no experimental, Hernandez-Sampieri y Mendoza (2018) la definen como, "estudios que se realizan sin la manipulación deliberada de variables y en los que solo se observan los fenómenos en su ambiente natural para analizarlos" (p. 175).

Esta investigación es de tipo descriptiva - correlacional de corte transeccional o transversal, puesto que se recolectan datos en un solo momento en el tiempo único. Se procedió al análisis de los resultados mediante el programa estadístico IBM SPSS Statistics 25. Para la recopilación de la información se utilizó la técnica de la encuesta utilizando escala tipo Likert con cinco alternativas de respuesta: siempre, casi siempre, algunas veces, casi nunca, nunca, correspondiéndole a cada respuesta un puntaje desde (05) puntos hasta (01) punto. Se seleccionó una muestra probabilística de 55 docentes de academias Pamer. 


\section{RESULTADOS}

La dimensión dirección está constituida por cinco indicadores. En la Tabla 1, se aprecian los resultados para cada indicador.

El indicador liderazgo producto de la aplicación de la encuesta arrojó como resultado una media ponderada de 2,25 que de acuerdo con la tabla de cálculos de Baremo diseñado para su interpretación, se ubicó en bajo nivel, asimismo, para el indicador relaciones interpersonales se aprecia una media ponderada de 3,14 que alcanzó la categoría de moderado nivel, del mismo modo, para el indicador motivación, la media ponderada fue 1,79 obteniendo la categoría de muy bajo nivel y en lo que respecta al indicador beneficios y recompensas el resultado fue de 1,53 alcanzando la categoría de muy bajo nivel. Finalmente, el indicador comunicación, la media ponderada fue de 2,42 , que obtuvo la categoría de bajo nivel.

Los porcentajes obtenidos por el nivel de escala para la dimensión de dirección son: nunca
$28,9 \%$, casi nunca $34,3 \%$, alguna vez $29,2 \%$, casi siempre 6,8\%, siempre $0 \%$. Tal como se observa en la Figura 1.

El resultado obtenido al aplicar el Rho de Spearman a nivel de la institución, arroja un resultado total de ,624; demostrando que existe una correlación positiva considerable entre Dirección y Desempeño Docente, lo que permite cubrir la expectativa planteada. En la Tabla 2, se aprecian los resultados.

\section{DISCUSIÓN}

Los resultados encontrados evidencian que la función de dirección tiene una correlación positiva con el desempeño profesional de los docentes en la institución de preparación preuniversitaria. La dimensión dirección presentó como resultado ponderado de sus cinco indicadores, un bajo nivel, obteniendo una media ponderada general de 2,16. D'Alessio (2015) al referirse a la importancia de la dirección señala que además de ser necesaria para mantener el rumbo y las estrategias para lograr el cumplimiento de

Tabla 1

\begin{tabular}{|c|c|c|c|c|c|c|c|c|c|c|c|c|c|c|}
\hline \multirow[t]{2}{*}{ Indicador } & \multirow[t]{2}{*}{ Item } & \multicolumn{2}{|c|}{$\begin{array}{c}1 \\
\text { Nunca }\end{array}$} & \multicolumn{2}{|c|}{$\begin{array}{c}2 \\
\text { Casi nunca }\end{array}$} & \multicolumn{2}{|c|}{$\begin{array}{c}3 \\
\text { Algunas veces }\end{array}$} & \multicolumn{2}{|c|}{$\begin{array}{c}4 \\
\text { Casi siempre }\end{array}$} & \multicolumn{2}{|c|}{$\begin{array}{c}5 \\
\text { Siempre }\end{array}$} & \multicolumn{2}{|c|}{ Total } & \multirow[t]{2}{*}{ Media } \\
\hline & & fa & $\mathbf{F r}$ & Fa & Fr & fa & $\mathbf{F r}$ & fa & Fr & fa & $\mathrm{Fr}$ & fa & $\mathrm{Fr}$ & \\
\hline \multirow{4}{*}{ Liderazgo } & 1 & 14 & $25.50 \%$ & 11 & $20.00 \%$ & 27 & $49.10 \%$ & 3 & $5.50 \%$ & 0 & $0.00 \%$ & 55 & $100 \%$ & \multirow{4}{*}{2.25} \\
\hline & 2 & 7 & $12.70 \%$ & 15 & $27.30 \%$ & 28 & $50.90 \%$ & 5 & $9.10 \%$ & 0 & $0.00 \%$ & 55 & $100 \%$ & \\
\hline & 3 & 23 & $41.80 \%$ & 18 & $32.70 \%$ & 14 & $25.50 \%$ & 0 & $0.00 \%$ & 0 & $0.00 \%$ & 55 & $100 \%$ & \\
\hline & 4 & 11 & $20.00 \%$ & 18 & $32.70 \%$ & 26 & $47.30 \%$ & 0 & $0.00 \%$ & 0 & $0.00 \%$ & 55 & $100 \%$ & \\
\hline \multirow{2}{*}{$\begin{array}{l}\text { Relaciones } \\
\text { interpersonales }\end{array}$} & 5 & 3 & $5.50 \%$ & 4 & $7.30 \%$ & 33 & $60.00 \%$ & 12 & $21.80 \%$ & 3 & $5.50 \%$ & 55 & $100 \%$ & \multirow{2}{*}{3.14} \\
\hline & 6 & 0 & $0.00 \%$ & 8 & $14.50 \%$ & 33 & $60.00 \%$ & 13 & $23.60 \%$ & 1 & $1.80 \%$ & 55 & $100 \%$ & \\
\hline \multirow[t]{4}{*}{ Motivación } & 7 & 22 & $40.00 \%$ & 25 & $45.50 \%$ & 8 & $14.50 \%$ & 0 & $0.00 \%$ & 0 & $0.00 \%$ & 55 & $100 \%$ & \multirow{4}{*}{1.79} \\
\hline & 8 & 8 & $14.50 \%$ & 19 & $34.50 \%$ & 12 & $21.80 \%$ & 14 & $25.50 \%$ & 2 & $3.60 \%$ & 55 & $100 \%$ & \\
\hline & 9 & 45 & $81.80 \%$ & 9 & $16.40 \%$ & 1 & $1.80 \%$ & 0 & $0.00 \%$ & 0 & $0.00 \%$ & 55 & $100 \%$ & \\
\hline & 10 & 27 & $49.10 \%$ & 27 & $49.10 \%$ & 1 & $1.80 \%$ & 0 & $0.00 \%$ & 0 & $0.00 \%$ & 55 & $100 \%$ & \\
\hline \multirow{3}{*}{$\begin{array}{l}\text { Beneficios y } \\
\text { recompensas }\end{array}$} & 11 & 32 & $58.20 \%$ & 21 & $38.20 \%$ & 2 & $3.60 \%$ & 0 & $0.00 \%$ & 0 & $0.00 \%$ & 55 & $100 \%$ & \multirow{3}{*}{1.53} \\
\hline & 12 & 46 & $83.60 \%$ & 7 & $12.70 \%$ & 2 & $3.60 \%$ & 0 & $0.00 \%$ & 0 & $0.00 \%$ & 55 & $100 \%$ & \\
\hline & 13 & 14 & $25.50 \%$ & 29 & $52.70 \%$ & 12 & $21.80 \%$ & 0 & $0.00 \%$ & 0 & $0.00 \%$ & 55 & $100 \%$ & \\
\hline \multirow[t]{4}{*}{ Comunicación } & 14 & 9 & $16.40 \%$ & 34 & $61.80 \%$ & 12 & $21.80 \%$ & 0 & $0.00 \%$ & 0 & $0.00 \%$ & 55 & $100 \%$ & \multirow{4}{*}{2.42} \\
\hline & 15 & 6 & $10.90 \%$ & 31 & $56.40 \%$ & 17 & $30.90 \%$ & 1 & $1.80 \%$ & 0 & $0.00 \%$ & 55 & $100 \%$ & \\
\hline & 16 & 0 & $0.00 \%$ & 8 & $14.50 \%$ & 30 & $54.50 \%$ & 16 & $29.10 \%$ & 1 & $1.80 \%$ & 55 & $100 \%$ & \\
\hline & 17 & 3 & $5.50 \%$ & 37 & $67.30 \%$ & 15 & $27.30 \%$ & 0 & $0.00 \%$ & 0 & $0.00 \%$ & 55 & $100 \%$ & \\
\hline \multicolumn{2}{|l|}{ Promedio } & 16 & $28.90 \%$ & 19 & $34.30 \%$ & 16 & $29.20 \%$ & 4 & $6.80 \%$ & 0 & $0.00 \%$ & 55 & $100 \%$ & \\
\hline
\end{tabular}

Nota: $\mathrm{Fa}=$ frecuencia absoluta; $\mathrm{Fr}$ = frecuencia relativa.

Elaboración propia (2020). 


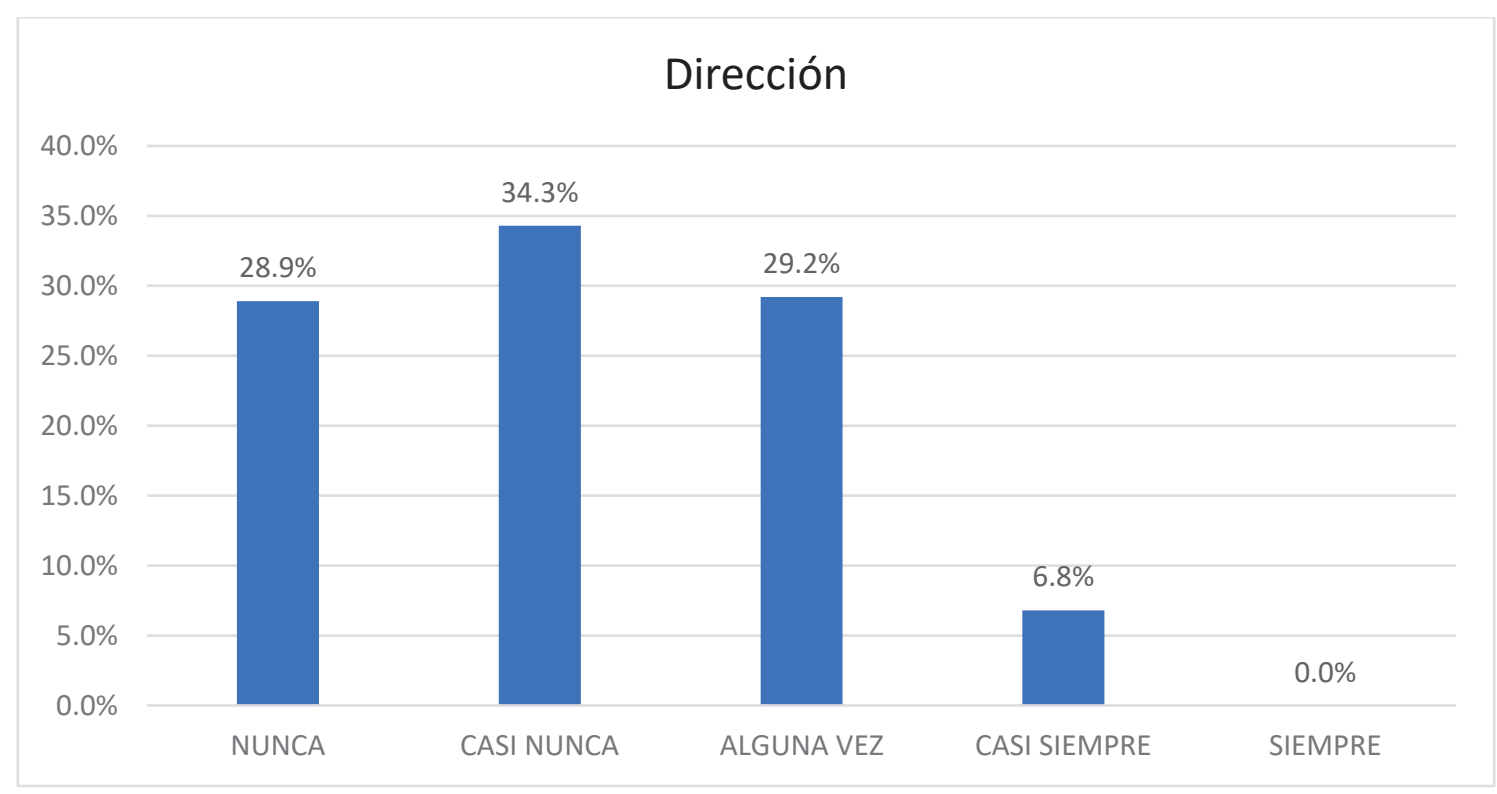

Figura 1. Porcentajes obtenidos por nivel de escala para la dimensión dirección. Elaboración propia (2020).

Tabla 2

Correlación Dirección y Desempeño Docente

\begin{tabular}{|c|c|c|c|c|}
\hline & & & Desempeño & Dirección \\
\hline \multirow[t]{6}{*}{ Rho de Spearman } & Desempeño & Coeficiente de correlación & 1,000 &, $624^{* *}$ \\
\hline & & Sig. (bilateral) & . & 000 \\
\hline & & $\mathrm{N}$ & 55 & 55 \\
\hline & Dirección & Coeficiente de correlación & ,624* & 1,000 \\
\hline & & Sig. (bilateral) & 000 & . \\
\hline & & $\mathrm{N}$ & 55 & 55 \\
\hline
\end{tabular}

**. La correlación es significativa en el nivel 0,01 (bilateral).

Nota: Elaboración propia basado en el programa estadístico IBM SPSS (2020).

la misión de la organización, esta función busca aumentar la productividad como vehículo para incrementar las posibilidades de competir con éxito.

En cuanto al liderazgo como elemento o factor humano utilizado en la dirección, supone seguidores y las personas tienden a seguir a los que ofrecen medios para satisfacer sus necesidades, anhelos y deseos, es comprensible que dirigir suponga motivar, estilos y enfoques de liderazgo y comunicación (Koontz et al., 2012). Los indicadores más críticos por encontrarse en muy bajo nivel, en esta dimensión se refieren a la motivación y a los beneficios y recompensas, lo que se traduce en un ambiente de trabajo para los docentes poco inspirador perjudicando la creatividad, la productividad y la excelencia educativa que declara en su misión la institución. Méndez (2018) sostiene que las empresas exitosas fundamentan su cultura organizacional en modelos humanistas de administración; donde se reconoce y da importancia a las personas en el ser. En este sentido, la motivación es ejercida por los líderes, quienes crean condiciones mediante estímulos salariales y económicos, al igual que con recompensas sociales simbólicas y no materiales.

Otro factor sumamente importante en toda institución es la comunicación y este elemento fue evaluado en la categoría de bajo nivel. Refiriéndose a la comunicación interna Fischman (2018) indica que, las personas necesitan siempre más información, se debe comunicar para evitar confusión o malentendidos. 
La dirección es una de las funciones principales de toda institución y está orientada a dirigir $\mathrm{y}$ facilitar las actividades dentro y fuera de esta. Para que el desempeño del docente mejore respecto al indicador dirección es importante tener en cuenta el liderazgo factor humano de dirección y cambio, la motivación, las relaciones interpersonales, la comunicación y el reconocimiento.

\section{CONCLUSIONES}

La dirección institucional sí se relaciona con el desempeño de los docentes en la institución de preparación preuniversitaria, así lo señala el resultado obtenido al aplicar el Rho de Spearman a nivel de la institución, donde arrojó un valor de ,624; demostrando que existe una correlación positiva considerable, por lo tanto, es importante que la institución fortalezca el rol de liderazgo de sus directores para superar la debilidad encontrada en este factor clave de competitividad. A su vez, realizar programas de recompensas dirigidos a reconocer los logros de sus docentes, así como incrementar sus beneficios socioeconómicos. Al mejorar estos dos factores, la motivación se incrementará en sus docentes favoreciendo así la dedicación y productividad académica, asimismo el realizar actividades de integración social, cultural, deportiva u otras logrará fortalecer las relaciones interpersonales. En cuanto a la comunicación, la institución debe utilizar diferentes recursos y estrategias, como reuniones periódicas, boletines informáticos (físicos y virtuales) y sistemas organizados de información, así como aplicar mecanismos efectivos para socializar y lograr que sus documentos estratégicos constituidos por la visión, misión, valores y objetivos sean conocidos y compartidos por todos sus trabajadores y de manera especial por sus docentes quienes representan su principal recurso. De igual manera, diseñar y poner en ejecución programas de adiestramiento permanente para fortalecer las competencias académicas, culturales y sociales del personal docente.

\section{REFERENCIAS BIBLIOGRÁFICAS}

Arbaiza, L. (2010). Comportamiento Organizacional: bases y fundamentos. Buenos Aires, Argentina: Cengage Learning.

Betancourt, J. (2019). La Responsabilidad Social como Fundamento para la Estrategia Comunicacional de Organizaciones Innovadoras.
Gestión en el Tercer Milenio, 22(43), 77-82. Recuperado de: https://revistasinvestigacion. unmsm.edu.pe/index.php/administrativas/article/view/16956

Chávez, M. (2017). Desempeño docente según estudiantes del VII ciclo de instituciones educativas técnicas del distrito de Pangoa - Satipo. (Tesis maestría). Universidad Nacional del Centro del Perú. Perú. Recuperado de: http://repositorio. uncp.edu.pe/handle/UNCP/4275

Chiroque, S. (2006). Discurso y nociones sobre el desempeño docente: Diálogo con maestros. Hacia una propuesta de criterios de buen desempeño docente. Lima, Perú: Consejo nacional de educación/Fundación SM, 2011. p. 65.

D’Alessio, F. (2015). El proceso estratégico: un enfoque de gerencia. (3era Ed.). Lima, Perú: Pearson Educación.

Díaz, M. (2006). La difícil tarea de educar. Madrid, España: Bruño. p. 87.

Fischman, D. (2018). Cuando el liderazgo no es suficiente. Lima, Perú: Editorial Planeta.

Hernández, R., Fernández, C. y Batista, P. (2006). Metodología de la Investigación. México: Mc Graw Hill.

Hernandez-Sampieri, R. y Mendoza, Ch. (2018). Metodología de la investigación. Las rutas cuantitativa, cualitativa y mixta. México: Mc Graw Hill.

Koontz, H., Wrihrich, H. y Cannice, M. (2012). Administración. Una perspectiva global y empresarial. (14aㅡ Edición). México: Editorial Mc Graw Hill.

Louffat, E. (2015). Administración: Fundamentos del proceso administrativo. (4ta edición). Buenos Aires. Argentina: Cengage Learning.

Marciaga, C. (2015). Importancia de la dirección en las organizaciones. Emprendices. Recuperado de: https://www.emprendices.co/importancia-de-la-direccion-en-las-organizaciones/

Méndez, C. (2018). Cultura y Clima: fundamentos para el cambio en la organización. Bogotá. Colombia: Editorial Alfaomega.

Ministerio de Educación (2014). Ley universitaria N³0220 art 100. Recuperado de: http://www. minedu.gob.pe/reforma-universitaria/pdf/ ley_universitaria.pdf

Montenegro, I. (2007). Evaluación del Desempeño. Docente Fundamentos, Modelos e Instrumentos. Bogotá, Colombia: Cooperativa Editorial Magisterio. p 31. 
Reyes, N. (2012). Liderazgo directivo y desempeño docente en el nivel secundario de una institución educativa deVentanilla-Callao. (Tesis maestría). USIL. Recuperado de: http://repositorio.usil. edu.pe/bitstream/123456789/1302/1/2012_ Reyes_Liderazgo $\% 20$ directivo $\% 20$ y\%20desempe $\%$ C3\%B10\%20docente $\% 20$ en $\% 20$ el $\% 20$ nivel $\% 20$ secundario $\% 20 \mathrm{de} \% 20$ una $\% 20$ instituci\%C3\%B3n\%20educativa\%20de\%20Ventanilla-\%20Callao.pdf

Rodríguez, M. (2017). El desempeño docente y el logro de aprendizaje en el área de comunicación en las instituciones educativas de Chaclacayo en el 2015. (Tesis maestría). Universidad Nacional Mayor de San Marcos. Perú. Recuperado de: https://cybertesis.unmsm.edu.pe/handle/20.500.12672/7287
Organización de las Naciones Unidas para la Educación, la Ciencia y la Cultura (2013). Situación Educativa de América Latina y el Caribe: Hacia la educación de calidad para todos al 2015. Santiago, Chile: Salesianos. p. 25. Recuperado de: http://www.unesco.org/new/fileadmin/MULTIMEDIA/FIELD/Santiago/images/SITIED-espanol.pdf 
